\title{
Association between Personality Traits and Metacognition among Pharmacy Students: Implication for Pharmaceutical Education
}

\author{
Xianmin Hu, Jun Wang*, Juan Liu, Yuhang Yao \\ Department of Pharmacy, College of Medicine, Wuhan University of Science and Technology, Wuhan, CHINA.
}

\begin{abstract}
Background: The importance of pharmacy practitioners' personality traits in their professional development and future patient-centered pharmaceutical care practice has been widely recognized. Metacognitive skills in critical thinking, self-directed learning as a critical part of pharmacy training has been emphasized in the new pharmacy education accreditation standard. Correlation between metacognition and personality has been demonstrated in patients with personality pathology. Objectives: This work aimed to study the association between personality and metacognition among Chinese pharmacy students. Materials and Methods: A total of 130 pharmacy students voluntarily completed the 30-item Metacognitions Questionnaire (MCQ-30) and NEO Five-Factor Inventory (NEO-FFI) to measure their metacognitions and personality traits, respectively. Results: Pearson Correlations showed a strong association between metacognition and NEO-FFI conscientiousness personality trait. NEO-FFI neuroticism was found weakly but significantly positively associated with overall MCQ-30 scores, strongly positively associated with metacognitive factors "Negative beliefs about worry" and "Cognitive self-consciousness", moderately negatively associated with "Positive beliefs about worry" or "Cognitive confidence" subscales of MCQ-30. Scores on the NEO-FFI extraversion, openness or agreeableness and the metacognitive factor "Positive beliefs about worry", "Cognitive confidence" or "Negative beliefs about worry" were highly correlated. Conclusion: This study firstly linked pharmacy students' personality traits to their metacognition levels, suggesting that educational interventions to develop metacognitive skills might contribute to the improvement of students' personality traits.
\end{abstract}

Key words: Personality, Metacognition, Association, Pharmacy students, Pharmaceutical education.

\section{INTRODUCTION}

As the responsible provision of pharmacotherapy aiming to achieve desired outcomes that improve the life quality of patients, modern pharmaceutical care emphasizes the patient-centered practice. ${ }^{1,2}$ Therefore, pharmacy practitioners need to build direct personal, professional and responsible relationships with patients. $^{3}$ During the current pharmaceutical care practice, pharmacists must assume the multiple functions of a communicator, decision maker, life-long learner, caregiver, tutor and manager, etc. ${ }^{4}$ Moreover, along with the development of interprofessional team collaboration in health care, pharmacists should undertake effective communication in a therapeutic team, which includes other pharmacists as well as other professionals such as physicians and nurses. ${ }^{3}$ Therefore, in order to improve the pharmaceutical care implementation and maintain the good interpersonal communication that is essential in clinical therapeutic relationship, the personality predisposition of pharmacists is necessary. At present, the importance of pharmacy practitioners' personality traits in their professional development has been widely
Submission Date: 04-06-2020; Revision Date: 25-08-2020; Accepted Date: 18-12-2020

DOI: 10.5530/ijper.55.1.10 Correspondence: Dr. Jun Wang Department of Pharmacy, College of Medicine, Wuhan University of Science and Technology, Wuhan-430065, CHINA.

Phone no: +86-027-

87661513

Email id: wangjun@wust. edu.cn

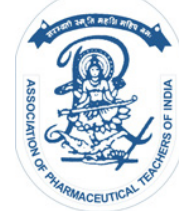

www.ijper.org 
recognized..$^{5-9}$ Rosenthal et al. ${ }^{7}$ linked pharmacists' personality traits to their behaviors and performance in the pharmacy practice research, an approach for the integration of new knowledge and practices. As individuals tend to choose the careers which match their personality, the congruence between the profiles of personalities and career choice among pharmacy professionals has been demonstrated. ${ }^{5,8}$ Their personality has been considered as a crucial factor in the professional paths taken by pharmacists. ${ }^{5,8} \mathrm{~A}$ pharmacist tend to actively engage in clinical practice when his/ her personality is in full coordination with his/her work environment. Otherwise, he/she would exhibit dissatisfaction, could not show effective behavior and be possibly limited in making a positive contribution to his/ her practice and work environment. ${ }^{5,8}$ The results from an online survey of second-year Doctor of Pharmacy students ${ }^{9}$ showed certain personality traits among future pharmacists influenced their preferences for health outcomes in the context of hypothetical cancer treatment decision-making. Thoresen et al. ${ }^{6}$ Showed the significant correlation between the personality profiles and the sales performance of pharmaceutical company sales representatives. Considering these uncovered correlation between pharmacy professionals' personality characteristics and work-related performance, successes, or career anchors, some educators have proposed the need for the training tailored specifically to the students' personality traits that are conducive to patient-centered pharmaceutical care practice.,10 However, because personality is believed to show considerable continuity over time, ${ }^{10}$ it might be difficult to change students' personality traits through the professional education during college.

From the perspective of psychology, accumulative evidences have revealed weak metacognition is closely associated with the severity of personality pathology. ${ }^{11-13}$ Accordingly, psychological interventions based on metacognitive training have been demonstrated to be effective in improving personality disorder symptoms, interpersonal and social functioning. ${ }^{14,15}$ Metacognition simply defined as the capacity to think about one's thinking is one's “knowledge and cognition about cognitive phenomena as well as monitoring of one's own memory, comprehension and cognitive enterprises". ${ }^{16,17}$ A person possessing high metacognitive skills is someone who can regulate his/her thinking and learning through selfassessment. Considering the ever-changing nature of the pharmacy profession, which requires ongoing selfassessment of knowledge and skills as well as life-long learning, the importance of metacognition skill training in pharmaceutical education has been highlighted in recent years. ${ }^{16-23}$

During the learning process, pharmacy students need metacognitive skills to supervise and adjust their thinking, understanding and reasoning and problem-solving, that are underlying components or desired outcomes of pharmaceutical curricula. Metacognition is essentially a means to address "what" "when" and "how" to learn. If a learner know what he/she knows and does not know, he/she will focus on acquiring the knowledge he/she is lacking. As for the pharmacy practitioners such as pharmacists, metacognitive skills may affect their professional judgments, for example, being met cognitively aware can lead to better critical thinking and monitoring of actions, thus prevent medication errors in the clinical settings. ${ }^{16-23}$ Considering the well-accepted role of metacognition in professional development, the new pharmacy education accreditation standard emphasized the metacognitive skills in critical thinking, self-directed learning as a critical part of pharmacy training. ${ }^{16-23}$

Metacognitive skills are useful for learners to develop effective self-directed, lifelong learning strategies and increase self-awareness, the latter of which is a vital component of professional and personal growth for pharmacy students. ${ }^{22}$ Self-awareness training has been shown to affect personality traits in healthy volunteers. ${ }^{24}$ More importantly, metacognition is fortunately not an innate skill, but can be effectively developed when certain educational approaches are employed. ${ }^{16-23}$ Therefore, if there is a close relationship between metacognition and personality traits among pharmacy students, it is encouraging that teaching metacognitive skills might be a pathway to develop students' personality traits, enable the student to be a better learner and become a better pharmacy professional. However, current evidences on the correlation between metacognition and personality are from patients with personality pathology. ${ }^{11-13}$ This work aimed to study the association between personality and metacognition among Chinese pharmacy students.

\section{MATERIALS AND METHODS \\ Student participants}

A total of 130 pharmacy students (53 males and 77 females; 35 first-year students, 30 students in their second year, 30 studying in the third-year of the program and 35 fourth graders) who were recruited via the Internet voluntarily completed two questionnaires to measure their personality traits and metacognitions, respectively, on June 4-5, 2019. Approval was received 
from the Ethics Committee of Medicine College, Wuhan University of Science and Technology (2019051).

\section{Measures}

Personality traits of student participants were measured with an instrument named the NEO Five-Factor Inventory (NEO-FFI or the Big Five), ${ }^{5-7,9}$ a 60 -item questionnaire with 5 -point Likert scales (from 1 point $=$ "strongly disagree" to 5 points $=$ "strongly agree") widely applied to evaluate features of 5 personality traits (i.e. neuroticism, extraversion, openness, agreeableness, conscientiousness).

The Metacognition Questionnaire-30 (MCQ-30) ${ }^{16}$ was applied to evaluate students' metacognition. As a 30-item questionnaire with 4-point Liker-type scale (from 1 point $=$ "do not agree" to 4 points $=$ "agree very much"). There are 5 subscales in MCQ-30 including "Positive beliefs about worry", "Negative beliefs about worry", "Cognitive confidence", "Need to control thoughts" and "Cognitive self-consciousness".

The bilingual versions in both English and Chinese of these two survey instruments were used to ensure the accurate comprehension of student respondents.

\section{Data analysis}

Statistical analyses were performed using SPSS version 22.0 (IBM Corp, Armonk, NY, USA). The association between MCQ-30 subscales or total scale and each of 5 personality traits was measured with Pearson's Correlation Coefficient (r). The gender or grade difference was determined using analysis of variance (ANOVA). When $p<0.05$, statistically significance was considered.

\section{RESULTS}

Mean scores and standard deviations on the NEOFFI as well as MCQ-30 scores are presented in Table 1. Our analysis revealed no significant gender or grade difference in NEO-FFI and MCQ-30 scores.

Importantly, as shown in Table 2 and Figure 1, analysis on correlations between NEO-FFI and MCQ-30 scores showed a significant correlation between total scores of MCQ-30 and NEO-FFI neuroticism ( $\mathrm{r}: 0.172, p<0.05)$, as well as a strong positive correlation between total scores of MCQ-30 and NEO-FFI conscientiousness (r:0.447, $p<0.01$ ).

As for the MCQ-30 subscales, as shown in Table 2 and Figure 2, NEO-FFI neuroticism showed the strongest positive correlation of "Negative beliefs aboutworry" (r:0.842, $p<0.01$ ), followed by "Cognitive self-consciousness" ( $\mathrm{r}: 0.311$, $p<0.01)$. Moderate negative correlation was found between NEO-FFI neuroticism and "Positive beliefs about

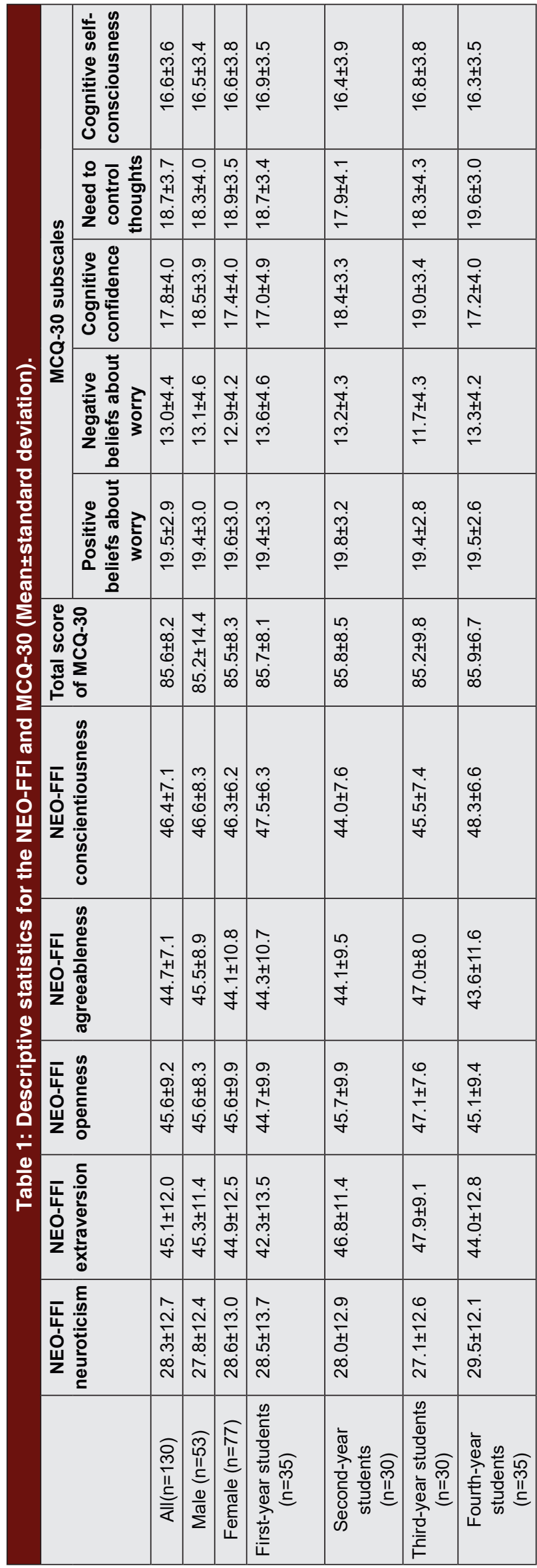



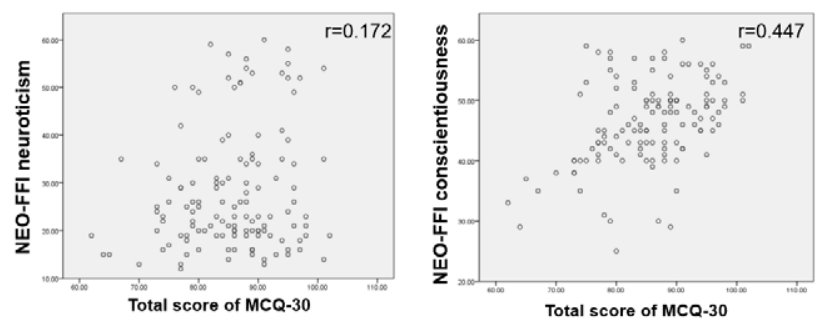

Figure 1: Pearson correlations between NEO-FFI neuroticism or conscientiousness and total scores of MCQ-30 of pharmacy student participants resulted significant correlations.

worry" (r:-0.572, $p<0.01)$ or "Cognitive confidence" (r:-0.590, $p<0.01)$ subscale of MCQ-30. NEO-FFI extraversion was strongly positively associated with "Positive beliefs about worry" (r:0.614, $p<0.01)$ or "Cognitive confidence" ( $\mathrm{r}: 0.705$, $p<0.01)$ and strongly negatively associated with "Negative beliefs about worry" (r: -0.622, $p<0.01$; Table 2 and Figure 3). A moderate positive correlation between NEO-FFI openness or agreeableness and "Positive beliefs about worry" or "Cognitive confidence" (r: 0.439-0.556, $p<0.01$ ) as well as a moderate negative correlation between NEO-FFI openness or agreeableness and "Negative beliefs about worry" (r:-0.558-0.583, $p<0.01)$ were shown in Table 2, Figure 4 and 5 . And a strong and positive correlation was obtained between NEO-FFI conscientiousness and "Need to control thoughts" (r:0.645, $p<0.01$; as in Table 2 and Figure 6)

\section{DISCUSSION}

In the field of psychopathology, a clear relationship between metacognitive deficits and personality pathology has been found in patients with personality disorders. ${ }^{11-15}$ For example, borderline personality disorder (BPD) patients showed bias in their metacognitive awareness of self-regulatory ability. Low metacognitive ability was closely related with more frequent presence of suspicious, paranoid and strange thoughts, ${ }^{11}$ as well as the higher expression of anger. ${ }^{14}$ Accordingly, metacognitive training or therapy which targets metacognitive mastery has

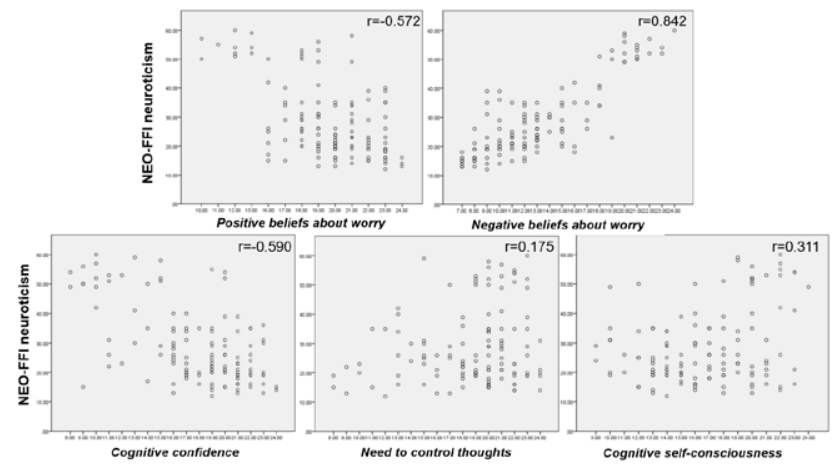

Figure 2: Pearson correlations between NEO-FFI neuroticism and MCQ-30 subscales of pharmacy student participants resulted significant correlations.

been considered as an useful add-on intervention for patients. Unlike these previous studies on patients with personality disorders, the present study is the first investigation uncovering the association between personality and metacognition among students. But in line with the findings from the personality disorder patients, ${ }^{11-15}$ this study suggested a possible interaction between pharmacy students' metacognitive ability and their personality traits during the undergraduate years. Based on this finding, it can be speculated that a metacognitive improvement during pharmaceutical education would consequently contribute to a general improvement in the students' personality traits, the latter of which has been well-accepted to play an important role in pharmacy practitioners' professional development as well as modern pharmaceutical care.-9

The MCQ-30 applied in this study is a widely used multidimensional measure for metacognitive beliefs and processes. ${ }^{16}$ The reliability of MCQ-30 as a measurement tool for metacognition has been supported in previous studies. ${ }^{16,17}$ Higher MCQ-30 scores indicate greater metacognitive activity. 16,17 In the present study, the MCQ-30 results were compared with the personality trait levels measured by the NEO-FFI, a validated and reliable instrument based on the five-factor model (FFM) of personality, which describes the universality

\begin{tabular}{|c|c|c|c|c|c|}
\hline \multicolumn{2}{|c|}{ Table 2: Pearson Correlation of MCQ-30 with the NEO-FFI. } \\
\hline MCQ-30 & $\begin{array}{c}\text { NEO-FFI } \\
\text { neuroticism }\end{array}$ & $\begin{array}{c}\text { NEO-FFI } \\
\text { extraversion }\end{array}$ & $\begin{array}{c}\text { NEO-FFI } \\
\text { openness }\end{array}$ & $\begin{array}{c}\text { NEO-FFI } \\
\text { agreeableness }\end{array}$ & $\begin{array}{c}\text { NEO-FFI } \\
\text { conscientiousness }\end{array}$ \\
\hline Total score & $0.172^{*}$ & 0.051 & 0.096 & 0.139 & $0.447^{* *}$ \\
\hline Positive beliefs about worry & $-0.572^{* *}$ & $0.614^{* *}$ & $0.529^{* *}$ & $0.482^{* *}$ & $0.172^{*}$ \\
\hline Negative beliefs about worry & $0.842^{* *}$ & $-0.622^{* *}$ & $-0.583^{* *}$ & $-0.558^{* *}$ & 0.080 \\
\hline Cognitive confidence & $-0.590^{* *}$ & $0.705^{* *}$ & $0.556^{* *}$ & $0.439^{* *}$ & 0.054 \\
\hline Need to control thoughts & $0.175^{*}$ & -0.120 & -0.017 & -0.020 & $0.645^{* *}$ \\
\hline Cognitive self-consciousness & $0.311^{* *}$ & $-0.289^{* *}$ & -0.107 & 0.129 & 0.053 \\
\hline
\end{tabular}

${ }^{*} p<0.05^{* *} p<0.01$ 

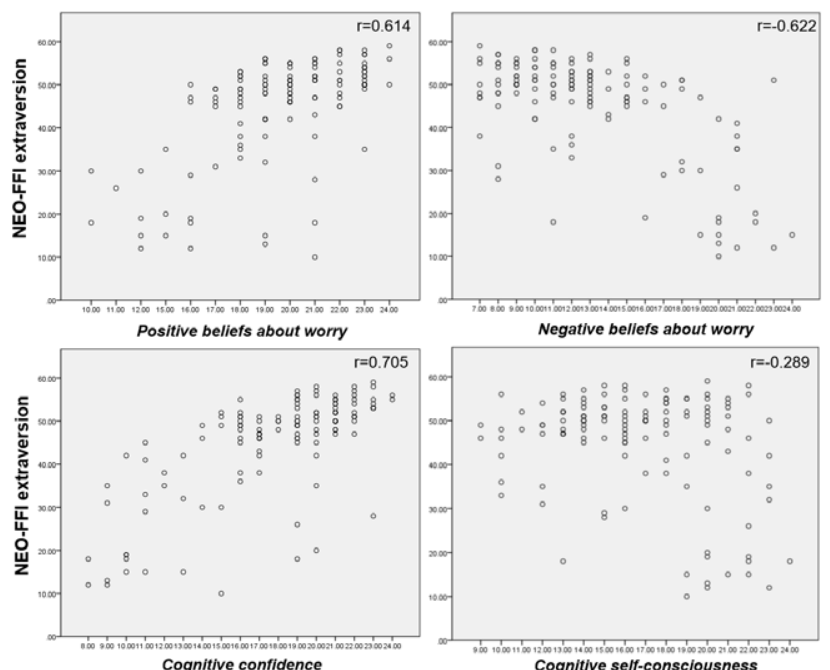

Figure 3: Pearson correlations between NEO-FFI extraversion and MCQ-30 subscales of pharmacy student participants resulted significant correlations.

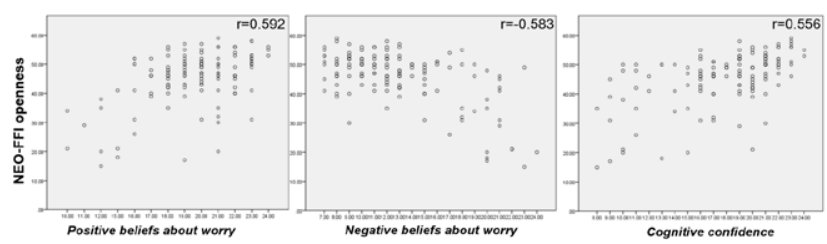

Figure 4: Pearson correlations between NEO-FFI openness and $M C Q-30$ subscales of pharmacy student participants resulted significant correlations.
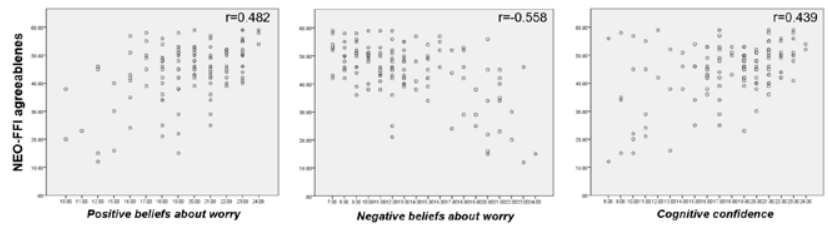

Figure 5: Pearson correlations between NEO-FFI agreeableness and MCQ-30 subscales of pharmacy student participants resulted significant correlations.

of personality and has been well-accepted among personality psychologists. ${ }^{5-7,9}$ Here, we reported a strong positive correlation between overall MCQ-30 scores and NEO-FFI conscientiousness among pharmacy students, which was evaluated using Pearson correlation. Accordingly, NEO-FFI conscientiousness also had a positive and strong correlation with the MCQ-30 subscale "Need to control thoughts". As one of personality dimensions included in five-factor personality model, conscientiousness is conceptually defined as the capacity to overcome impulses to act in a goal- and task-directed manner. ${ }^{3,9}$ Importantly, conscientiousness trait has been considered as a key factor in treatment-related outcome preferences among pharmacy students. ${ }^{3,9}$ Our results suggested that pharmacy students with greater

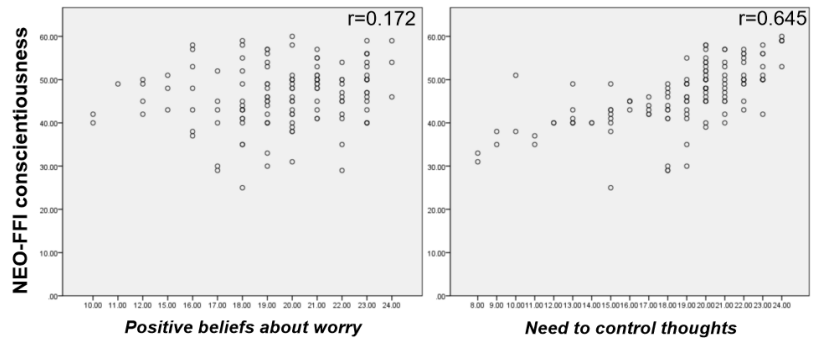

Figure 6: Pearson correlations between NEO-FFI conscientiousness and MCQ-30 subscales of pharmacy student participants resulted significant correlations.

metacognitive activity, in particular, with metacognitive beliefs concerning the need to control their own thoughts, tend to possess higher individual's organizational ability. In other words, conscientiousness is a positive predictor for a pharmacy student' metacognitive ability. A student who can better regulate his/her thinking and learning through self-assessment appeared to be characterized by a higher intensity of conscientiousness.

There was no strong correlations between overall MCQ30 scores and the other four personality dimensions (neuroticism, extroversion, agreeableness or openness) involved in FFM. However, NEO-FFI neuroticism was found weakly but significantly positively associated with overall MCQ-30 scores, strongly positively associated with metacognitive factors "Negative beliefs about worry" and "Cognitive self-consciousness", moderately negatively correlated to "Positive beliefs about worry", "Cognitive confidence" subscales in MCQ-30. These results suggest that a pharmacy student who has higher difficulty in coping with stress tends to have more beliefs regarding uncontrollability and risk, focus more attentions on thought processes, but have less confidence in attention and memory. NEO-FFI extraversion means the intensity and quantity of interpersonal interactions. ${ }^{3,9} \mathrm{NEO}-\mathrm{FFI}$ openness refers to a person's interest in discovering the world and his/her attitude regarding new ideas. ${ }^{3,9}$ NEO-FFI agreeableness is conceptually described as cooperation, altruism and willingness to exhibit kindness and warmth. ${ }^{9}$ In our study, the scores on the NEO-FFI extraversion, openness or agreeableness and the metacognitive factor "Positive beliefs about worry", "Cognitive confidence" or "Negative beliefs about worry" were highly correlated, which suggests that these three personality traits was associated with the metacognitive beliefs and confidence.

So far, pharmaceutical educators have employed some teaching strategies for the improvement of students' metacognition. ${ }^{16-23}$ In a review conducted by Medina et al. ${ }^{16}$ interventions including exam reviews, cognitive apprenticeships, thinking out loud protocols, modeling 
of metacognitive skills, self-explanation methods, reflection assignments and judgment of understanding assignments have been suggested to be implemented in the didactic setting to help students improve metacognitive skills in the classroom. In the experiential setting, teachers can raise students' self-awareness and help metacognitive thinking to occur automatically through emphasizing mastery goal setting, requesting clinical documentation with an explanation, using questioning and feedback techniques and scaffolding experiential rotation structure, etc. Based on the correlation between metacognition and personality which was reported in our study, these metacognitive training strategies might also be useful for improving the students' personality traits that are conducive to their professional development and future patient-centered pharmaceutical care practice.

There are some limitations in the present study. Further empirical supports on the effectiveness of metacognitive training in personality improvement during pharmaceutical education are needed. Moreover, during the survey, a total of 229 pharmacy students were enrolled in the studied university. Because the student respondents were recruited via the Internet voluntarily, the overall response rate was $57 \%$. To verify our findings, larger samples should be included in further researches.

\section{CONCLUSION}

This is the first study that linked pharmacy students' personality traits to their metacognition levels. We observed a strong association between metacognition and conscientiousness personality trait. The other four personality dimensions (neuroticism, extroversion, agreeableness or openness) involved in FFM also showed varying degrees of association with some metacognitive factors. These results suggested that educational interventions to develop metacognitive skills might contribute to the improvement of students' personality traits, the latter of which has been well-accepted to be important for their professional development and future patient-centered pharmaceutical care practice.

\section{ACKNOWLEDGEMENT}

This research has been financially supported by the medical education research project from Medical education branch of the Chinese Medical Association and medical education professional committee of the Chinese Association of Higher Education (2018BN02084), the educational science planning project in Hubei province (2019GB019), the Provincial Teaching Research Project in Hubei (2014230) and Teaching
Research Project in Wuhan University of Science and Technology (2017X057).

\section{CONFLICT OF INTEREST}

The authors declare that there are no conflicts of interest.

\section{ABBREVIATIONS}

ANOVA: Analysis of Variance; BPD: Borderline Personality Disorder; FFM: Five-factor Model; MCQ-30: Metacognition Questionnaire-30; NEO-FFI: NEO Five-Factor Inventory.

\section{REFERENCES}

1. Moltó-Puigmartí C, Vonk R, Ommeren GV, Hegger I. A logic model for pharmaceutical care. J Health Serv Res Policy. 2018;23(3):148-57.

2. Wolters M, Hulten RV, Blom L, Bouvy ML. Exploring the concept of patient centred communication for the pharmacy practice. Int $\mathrm{J}$ Clin Pharm. 2017;39(6):1145-56.

3. Skrzypek M, Turska D, Marzec A, Szczygieł K. Personality traits and personal values as retail pharmacy choice predictors in the context of pharmaceutical care requirements. Res Social Adm Pharm. 2020;16(1):68-73.

4. Cordina M, Lauri MA, Lauri J. Patient-oriented personality traits of first-year pharmacy students. Am J Pharm Educ. 2010;74(5):84.

5. Hall J, Rosenthal M, Family H, Sutton J, Hall K, Tsuyuki RT. Personality traits of hospital pharmacists: Toward a better understanding of factors influencing pharmacy practice change. Can J Hosp Pharm. 2013;66(5):289-95.

6. Thoresen CJ, Bradley JC, Bliese PD, Thoresen JD. The big five personality traits and individual job performance growth trajectories in maintenance and transitional job stages. J Appl Psychol. 2004;89(5):835-53.

7. Rosenthal M, Sutton J, Austin Z, Tsuyuki RT. Relationship between personality traits and pharmacist performance in a pharmacy practice research trial. Can Pharm J. 2015;148(4):209-16.

8. Cordina M, Lauri MA, Lauri J. Career paths and personality in pharmacy. Int J Clin Pharm. 2012;34(6):876-84.

9. Law EH, Jiang R, Kaczynski A, Mühlbacher A, Pickard AS. The Role of Personality in Treatment-Related Outcome Preferences among Pharmacy Students. Am J Pharm Educ. 2019;83(7):6891.

10. Cordina M, Lauri MA, Buttigieg R, Lauri J. Personality traits of pharmacy and medical students throughout their course of studies. Pharm Pract. 2015;13(4):640.

11. Vega D, Torrubia R, Marco-Pallarés J, Soto A, Rodriguez-Fornells A. Metacognition of daily self-regulation processes and personality traits in borderline personality disorder. J Affect Disord. 2020;267:243-50.

12. Outcalt J, Dimaggio G, Popolo R, Buck K, Chaudoin-Patzoldt KA, Kukla M, et al. Metacognition moderates the relationship of disturbances in attachment with severity of borderline personality disorder among persons in treatment of substance use disorders. Compr Psychiatry. 2016;64:22-8.

13. Carcione A, Riccardi I, Bilotta E, Leone L, Pedone R, Conti L, et al. Metacognition as a Predictor of Improvements in Personality Disorders. Front Psychol. 2019;10:170.

14. Schilling L, Moritz S, Kriston L, Krieger M, Nagel M. Efficacy of metacognitive training for patients with borderline personality disorder: Preliminary results. Psychiatry Res. 2018;262:459-64.

15. Nordahl HM, Wells A. Metacognitive Therapy of Early Traumatized Patients with Borderline Personality Disorder: A Phase-II Baseline Controlled Trial. Front Psychol. 2019;10:1694.

16. Medina MS, Castleberry AN, Persky AM. Strategies for Improving Learner Metacognition in Health Professional Education. Am J Pharm Educ. 2017;81(4):78.

17. Hu X, Wang J, Chen L. Metacognitive Awareness of Third-Year Chinese Undergraduate Pharmacy Students in Wuhan University of Science and 
Technology: Preliminary Evaluation and Implications for Training of Life-long Learners. Indian J Pharm Educ. 2016;50(2):251-60.

18. Medina MS, Castleberry AN, Persky AM. Strategies for Improving Learner Metacognition in Health Professional Education. Am J Pharm Educ. 2017;81(4):78.

19. Steuber TD, Janzen KM, Walton AM, Nisly SA. Assessment of Learner Metacognition in a Professional Pharmacy Elective Course. Am J Pharm Educ. 2017;81(10):6034.

20. Chu Y, Palmer S, Persky AM. Assessing metacognition in the classroom: Student help-seeking behavior. Curr Pharm Teach Learn. 2018;10(11):1478-87.
21. Pate A, Lafitte EM, Ramachandran S, Caldwell DJ. The use of exam wrappers to promote metacognition. Curr Pharm Teach Learn. 2019;11(5):492-8.

22. Fox L, Piccorelli A, Bruch D. Practicing metacognition through the use of repeated posttest predictions. Curr Pharm Teach Learn. 2019;11(6):630-4.

23. Nisly SA, Sebaaly J, Fillius AG, Haltom WR, Dinkins MM. Changes in Pharmacy Students' Metacognition through Self-Evaluation During Advanced Pharmacy Practice Experiences. Am J Pharm Educ. 2020;84(1):7489.

24. Visani G, Venturi P, Isidori A, Tronconi S. Self-awareness training associated with personality traits and sex: A pilot study. Psychol Rep. 2010;107(1):318-20.
PICTORIAL ABSTRACT

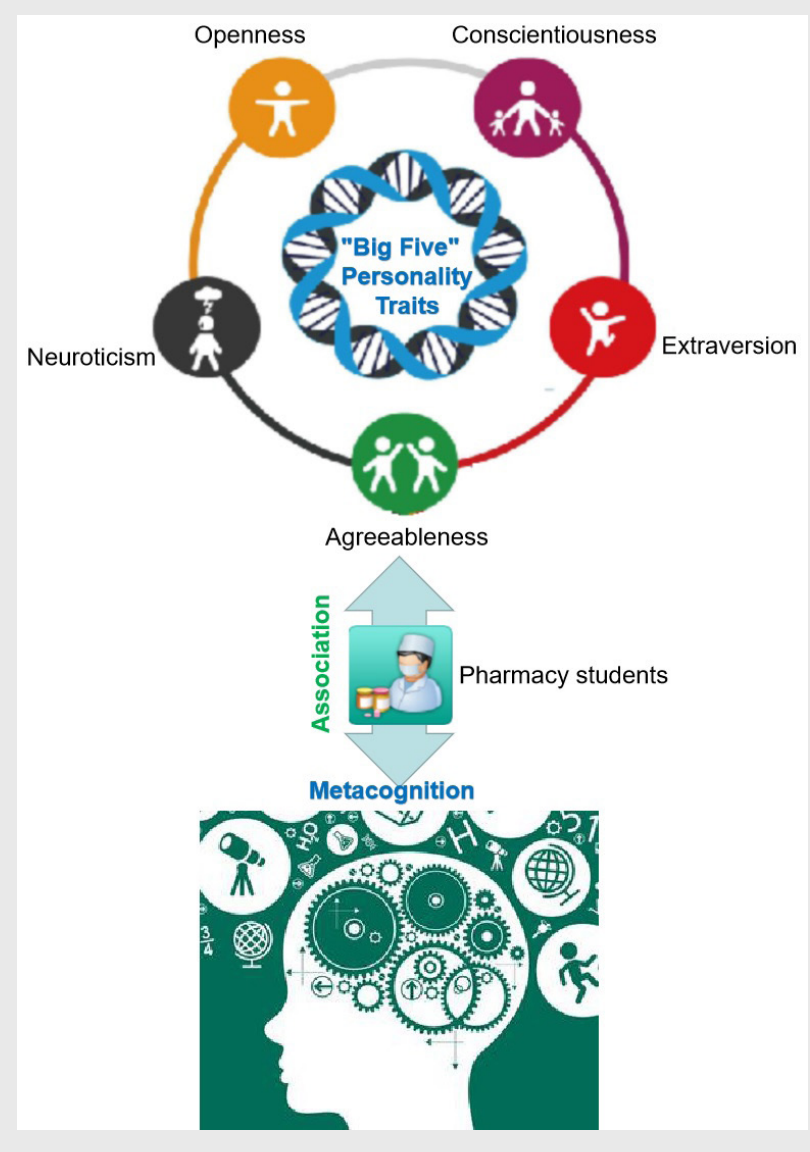

\section{SUMMARY}

- There is an association between pharmacy students'metacognitive ability and their personality traits during the undergraduate years.

- Hence, metacognitive training strategies might be useful for improving the students'personality traits that are conducive to their professional development and future patient-centered pharmaceutical care practice.

\section{About Authors}

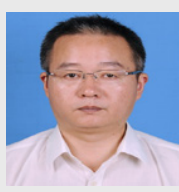

Xianmin $\mathrm{Hu}$ is a lecturer at the Department of Pharmacy, College of Medicine, Wuhan University of Science and Technology (Wuhan, China). He specializes in research on the higher Pharmaceutical Education

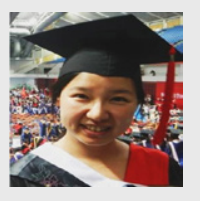

Jun Wang is an Associate Professor at the Department of Pharmacy, College of Medicine, Wuhan University of Science and Technology (Wuhan, China), received her Ph.D. from College of Pharmacy, Huazhong University of Science and Technology in 2013. She has projects in collaboration with the provincial institutions. Her research focused on the higher Pharmaceutical Education and Pharmacology.

Cite this article: $\mathrm{Hu}$ X, Wang J, Liu J, Yao Y. Association between Personality Traits and Metacognition among Pharmacy Students: Implication for Pharmaceutical Education. Indian J of Pharmaceutical Education and Research. $2021 ; 55(1): 70-6$. 